\title{
Efecto de la emoción sobre la memoria prospectiva: un nuevo enfoque basado en procedimientos operantes
}

\section{The effect of emotion on prospective memory: a new approach based on operant procedures}

\author{
Fernando Gordillo León ${ }^{1}$, José M. Arana Martínez ${ }^{1}$, \\ Juan J. G. Meilán ${ }^{1}$, Lilia Mestas Hernández ${ }^{2}$ \\ ${ }^{1}$ Departamento de Psicología Básica, Psicobiología y Metodología. Universidad de Salamanca (España), \\ ${ }^{2}$ Facultad de Estudios Superiores Zaragoza, Universidad Nacional Autónoma de México (México).
}

Disponible online 30 de diciembre de 2010

\begin{abstract}
La memoria prospectiva, definida como el recuerdo de realizar una acción programada en un momento determinado del futuro, puede verse afectada de diferente manera por el contenido emocional de los elementos que la determinan. En el contexto de laboratorio esta cuestión puede reproducirse mediante la inducción de emociones durante la realización de la tarea de memoria prospectiva. En este sentido, nos preguntamos si al inducir una emoción negativa o positiva tras la realización de la acción programada, estamos "castigando" y "reforzando", respectivamente, dicha conducta, y de ser así, qué efectos tendría sobre el recuerdo de volver a realizarla en un futuro. Discutimos este planteamiento a través del análisis de recientes trabajos que han abordado el tema desde diferentes puntos de vista, y proponemos la utilización de procedimientos operantes para analizar el efecto de la emoción sobre la memoria prospectiva.
\end{abstract}

Palabras Clave: castigo, emoción, memoria prospectiva, refuerzo.

Prospective memory denotes the ability to remember to perform actions in the future, and may be affected in different ways by the emotional content of the elements determining it. In the laboratory context, this situation can be reproduced through the induction of emotions during the performance of prospective memory tasks. We investigated whether by inducing a negative or positive emotion after the performance of a programmed task we were respectively "punishing" or "rewarding" such responses. If this proved to be the case, we further studied whether this would have an effect on remembering to perform it again in the future. We discuss this issue by analysing recent related work and we propose the use of operant procedures to analyze the effect of emotion on prospective memory.

Keywords: punishment, emotion, prospective memory, reinforcement.

Correspondencia: Fernando Gordillo León. Departamento de Psicología Básica, Psicobiología y Metodología, Universidad de Salamanca. Facultad de Psicología. Avda de la Merced 109-131. 37005 Salamanca (España). Teléfono: 662472038. E-mail: fgordilloleon@hotmail.com E-mail de los otros autores: José María Arana Martínez: arana@usal.es, Juan José García Meilán: meilan@usal.es, Lilia Mestas Hernández: lilia_mestas@yahoo.com.mx 
La memoria prospectiva (MP) está relacionada con el recuerdo de realizar una acción programada en un momento determinado del futuro (Kliegel, McDaniel y Einstein, 2008). Kvavilashvili $(1987,1992)$ propuso distinguirla de la memoria retrospectiva por cuanto requiere acordarse de recordar, "remembering to remember". La primera distinción entre memoria prospectiva y retrospectiva fue realizada por Meacham y Leiman (1982). Desde entonces, su delimitación conceptual se ha convertido en un requisito necesario para generar nuevas iniciativas de investigación (Graff y Uttl, 2001), así como para adecuar su análisis a los objetivos clínicos y diagnósticos presentes de manera recurrente en la literatura científica de los últimos años, que van desde la interacción entre la MP y diferentes procesos cognitivos (Basso, Ferrari y Palladino, 2010; McFarland y Glisky, 2009), hasta su relación con un heterogéneo grupo de patologías y tópicos de la problemática social -esquizofrenia (Chan et al., 2008; Twamley et al., 2008; Ungvari, Xiang, Tang y Shum, 2008; Wang et al., 2010); esclerosis múltiple (Adda, Castro, Além-Mar e Silva, de Manreza y Kashiara, 2008); Alzheimer (Livner, Laukka, Karlsson y Bäckman, 2009); Parkinson (Foster, McDaniel, Repovŝ y Hershey, 2009); SIDA (Woods et al., 2008); retraso mental (Meilán, Salgado, Arana, Carro y Jenaro, 2008); ingesta de alcohol (Heffernan, Clark, Bartholomew, Ling y Stephens, 2010); métodos anticonceptivos (Matter y Meier, 2008) -. Se conforma así, un marco teórico amplio y heterogéneo, donde la MP es analizada a diferentes niveles, y donde distintos procesos cognitivos participan en las fases que conforman el procesamiento de la intención.

Sin embargo, aún se conoce poco sobre cómo las emociones afectan a la memoria prospectiva (Kliegel et al., 2005). Resulta sorprendente comprobar cómo en el último congreso de memoria prospectiva celebrado en Vancouver (2010), tan sólo el $1.17 \%$ de los trabajos presentados trataban de manera directa la relación entre emoción y MP (tabla 1). Esto es debido, en parte, a que no ha recibido el mismo tratamiento metodológico que la memoria retrospectiva (e.g., Fernández-Rey y Redondo, 2007; Gordillo et al., 2010; Redondo y Fernández-Rey, 2010; Simón, Ruiz y Suengas, 2009), lo que ha impedido una mayor precisión en los resultados, y por lo tanto una mejor comprensión de los mismos. Es decir, no se ha tratado la emoción en sus niveles básicos de valencia y activación, lo que permitiría una inducción directa sobre los principales elementos que conforman la tarea de memoria prospectiva (tarea de fondo, claves de recuperación y conducta intencionada). Este planteamiento resulta lógico si pensamos que la emoción, a lo largo de nuestro quehacer diario, aparece de manera puntual con variaciones de intensidad y cualidad, y que su efecto sobre la tarea de fondo (e.g., las tareas propias de nuestro trabajo diario), las claves de recuperación (e.g., un anuncio de bebés que nos alerta de que debemos recoger a nuestros hijos del colegio) y la conducta intencionada (e.g., esperarlos al salir de clase), tendrá consecuencias diferentes sobre el recuerdo de realizar dicha conducta en el futuro.
Tabla 1. Temáticas relacionadas con la memoria prospectiva tratadas en el 3er Congreso Internacional de Memoria Prospectiva (Vancouver, 2010).

\begin{tabular}{lr} 
Enfermedades neurológicas & $20.00 \%$ \\
Trabajos con animales & $1.17 \%$ \\
Problemática social & $10.59 \%$ \\
Procesos cognitivos & $25.89 \%$ \\
Metodología & $12.94 \%$ \\
Medida & $3.53 \%$ \\
Envejecimiento & $21.18 \%$ \\
Neuroimagen & $3.53 \%$ \\
Emoción y Motivación & $1.17 \%$ \\
\hline
\end{tabular}

Utilizar un estado emocional constante para determinar su efecto sobre la MP resulta complicado de conseguir en tareas de laboratorio, por cuanto tiende a diluirse en el transcurso de la prueba (e.g., Kliegel et al., 2005), e imposible de mantener en la vida real. Pero además, carece de validez ecológica ya que no refleja el efecto que la fluencia de emociones cambiantes y adecuadas al entorno, ejerce sobre el proceso intencional (formación, retención y ejecución de la intención), que también se desarrolla en ese entorno, participando en la formación de dichas emociones (consecuencias negativas de olvidarse de recoger a los niños del colegio), y siendo influido por ellas, de forma que, el afecto negativo generado por la no realización de la tarea (olvidarse de recoger a los niños), incrementaría la probabilidad de que la próxima vez se recuerde (Arana et al., 2010).

La memoria prospectiva no es un tipo de memoria delimitado por conceptos claros y estructuras específicas, muy al contrario, participan diferentes procesos cognitivos y estructuras neurales, tal como se verá en el siguiente apartado. La emoción es parte de este complejo sistema de generación y cumplimiento de intenciones, y bien se estudie como procesos motivacionales o de manera más básica a través de sus componentes principales, se debe partir de una metodología que permita elaborar hipótesis y que amplíe, en definitiva, la curiosidad sobre el tema, que hasta la fecha no se ha generado, posiblemente por estas dificultades metodológicas.

Se abordará esta cuestión y sus derivaciones teóricas a través de los diferentes artículos que han tratado el tema, con el objetivo de proponer un nuevo enfoque de investigación que permita el estudio del efecto de la emoción sobre la MP de manera más precisa y controlada, lo que sin duda ayudaría a la comprensión de este proceso cognitivo y a su integración en el ámbito clínico.

\section{Memoria prospectiva}

El proceso de recordar y llevar a término una intención puede establecerse en cuatro fases (Glisky, 1996): 1) Codificación de la intención y planificación de la acción; 2) almacenamiento del plan por un intervalo de tiempo; 3) recuperación de la intención, y 4) realización de la acción y su recuerdo. 
Una distinción clásica en tareas de MP (Einstein y MacDaniel, 1996) es la que diferencia entre tareas basadas en tiempo (e.g., recordar apretar la tecla " 0 " cada 5 minutos), y tareas basadas en eventos (e.g., recordar apretar la tecla "0" cada vez que aparezca un triangulo en pantalla). Arana et al. (2010) determinan los elementos que componen una tarea de MP basada en eventos del siguiente modo, 1) tarea que los participantes deben realizar durante la prueba (tarea de fondo); 2) acción programada en las instrucciones que debe realizarse bajo determinadas circunstancias (conducta intencionada), y 3 ) estímulos que permiten el recuerdo de realizar dicha acción y que aparecen dentro de la tarea de fondo (claves de recuperación). Con esta clara estructuración metodológica es fácil comprender a simple vista las partes que componen una tarea de MP y su relación con las fases anteriormente comentadas.

En la ejecución de este tipo de tareas pueden estar involucrados una serie de procesos cognitivos (Meilán, Pérez y Arana, 2005), como son las funciones ejecutivas; la memoria de trabajo, episódica y retrospectiva; la atención; la autorregulación de la conducta y la motivación. Esta confluencia de procesos cognitivos parte de un conjunto de estructuras cerebrales que han sido propuestas como componentes del sustrato neural que permitiría la correcta formación, desarrollo y ejecución de la intención. En este sentido, es conocida la participación de las regiones frontales en la MP (Shallice y Burgess, 1991; Shimamura, Janowsky y Squire, 1991) y recientemente se han propuesto otras estructuras cerebrales, como las regiones basales (Umeda, Nagumo y Kato, 2006); las vías frontoestriales (Costa, Peppe, Caltagirone y Carlesimo, 2008; Foster et al., 2009); y el cortex prefrontal anterior (Reynolds, West y Braver, 2009; Simons, Scholvinck, Gilbert, Frith y Burgess, 2006).

De especial relevancia resulta la implicación de las funciones ejecutivas en la MP, y en concreto la memoria de trabajo. MacFarland y Glisky (2009), informan de que la MP se deteriora en la vejez debido a los déficit que el paso del tiempo provoca en las funciones ejecutivas, de lo que se deduce que la corteza prefrontal, como ejecutivo central, tiene un alto grado de participación. Sin embargo, determinados estudios parecen no evidenciar que el paso del tiempo perjudique de manera significativa la MP y esta falta de resultados es debida a que gran parte de estos trabajos se realizaron en el contexto de la vida real (e.g., pedir a los sujetos que llamen al experimentador a una hora determinada), donde es posible utilizar estrategias compensatorias que no serían posibles en tareas de laboratorio (Schmidt, Berg y Deelman, 2001). Este supuesto se confirmó en un meta-análisis realizado por Henry, MacLeod, Phillips y Crawford (2004), donde se concluye que en tareas de laboratorio, tanto basadas en eventos como en tiempo, los adultos jóvenes tienen un mejor rendimiento que los adultos mayores. Por otro lado, éstos tienen un mejor rendimiento en tareas realizadas en un contexto natural, hecho relacionado, tal como se apuntaba anteriormente, con el uso de estrategias de recuerdo consolidadas a lo largo de la vida.
El entrenamiento en la mejora de los procesos cognitivos que determinan el correcto funcionamiento de la MP, con el fin de ayudar en la rehabilitación de diferentes patologías o a la mejora en los déficit ocasionados por el paso del tiempo, puede considerarse un planteamiento indirecto, por cuanto no se establece una acción de mejora sobre la conducta a recordar (conducta intencionada), sino sobre las funciones cognitivas que permitirían dicho recuerdo. Resulta evidente que todo entrenamiento conlleva un incremento en la actividad de las regiones cerebrales relacionadas con la función cognitiva a mejorar. Este tipo de programas trata de habilitar los procesos que garantizan una correcta funcionalidad de la memoria prospectiva; es decir, estamos mejorando la atención, la memoria retrospectiva, las funciones ejecutivas, la motivación, para que llegado el momento se facilite el acceso al recuerdo de realizar una conducta programada. Pero resulta complicado establecer una clara causalidad entre el sustrato neurológico, la función cognitiva y la conducta resultante, dada la cantidad de elementos y niveles que confluyen y determinan la MP. Sin embargo, este tipo de programas no se aplican de manera aislada y es frecuente realizarlos junto a otros en los que el sujeto ejercita su memoria en el contexto de la vida diaria (véase Fish, Wilson y Manly, 2010). Por ejemplo, mediante el entrenamiento continuado de la memoria prospectiva en tareas sencillas (e.g., Sohlberg, White, Evans y Mateer, 1992), o con el aprendizaje de estrategias compensatorias (Fleming, Shum, Strong y Lightbody, 2005), que permitiría asociar la conducta intencionada (e.g., tomar una pastilla a las 3) con otra conducta habitual que, en cierto sentido, actúe como clave de recuperación (e.g., tomar un café).

En este complejo contexto se debe incorporar una variable más que no ha sido tratada con el merecido interés; nos referimos a la emoción y su efecto sobre la memoria prospectiva.

\section{Emoción y memoria prospectiva}

La emoción, en su relación con la MP, ha sido tratada relativamente poco. En la mayoría de los casos se cumple la intuición de que los estados emocionales negativos perjudican la MP (Kliegel y Jäger, 2006). En cuanto a las emociones positivas algunos estudios informan de que el estado de ánimo positivo incrementa los procesos de planificación, importantes para la correcta ejecución de la intención (Oaksfoard, Morris, Grainger, y Williams, 1996; Phillips, Smith y Gilhooly, 2002), mientras que para Kuhl y Kazén (1999), el afecto positivo facilitaría la ejecución de una conducta intencionada a través de una menor inhibición entre las intenciones almacenadas y sus sistemas de output motor.

Los métodos utilizados para analizar los efectos de la emoción sobre la MP son muy variados. Recientemente algunos trabajos han investigado la influencia de la emocionalidad de las claves de recuperación en la MP, reportándose resultados contradictorios. Por un lado se informa de que la valencia, posi- 
tiva o negativa, asociada a la claves de recuperación perjudica la MP a través de una disrupción en el procesamiento atencional (Clark-Foos, Brewer y Marsh, 2009) mientras que por otro lado se informa de una facilitación gracias a la mayor saliencia que la emoción proporciona a dichas claves (Altgassen, Phillips, Henry, Rendell y Kliegel, 2010). Estas diferencias advierten de la dificultad de inducir una emoción dentro de una tarea de MP, y de la necesidad de controlar de manera adecuada y con métodos de inducción emocional precisos y contrastados, dichas aplicaciones. Estos trabajos son relevantes, por cuanto abordan el tema de manera sustancialmente diferente.

La emoción ha sido analizada en la MP bajo diferentes prismas, como la ansiedad (Harris y Cumming, 2003; Harris y Menzies, 1999); la depresión (Altgassen, Kliegel y Martin, 2009; Rude, Hertel, Jarrold, Covich y Hedlund, 1999); el tipo de conducta intencionada (agradable o desagradable) (Kvavilashvili y Ellis, 1996; Meacham y Kushner, 1980); bajo situaciones estresantes (Schmidt, 2004), o como motivación, a través del efecto que los incentivos tienen sobre la realización de la conducta intencionada. Respecto a este último punto, Meacham y Singer (1977) encontraron que, efectivamente, la motivación era una variable crítica de este tipo de recuerdo en adultos sanos. Un incentivo moderado fue suficiente para incrementar el recuerdo prospectivo en una prueba en la que los participantes debían recordar enviar a tiempo una serie de tarjetas al experimentador. En el mismo sentido, Somerville, Wellman y Cultice (1983) encontraron que incluso niños de dos años son capaces de recordar tareas como "parar a comprar un helado" el $80 \%$ de las veces. Sin embargo, Guajardo y Best (2000) obtuvieron el resultado contrario. Analizaron el efecto del incentivo sobre el rendimiento en una tarea de memoria prospectiva en niños, no encontrando tales efectos. Es decir, el incentivo no produjo ningún efecto sobre el rendimiento. Meilán, Pérez, Arana y Valles (2006) encontraron que el incentivo no mejora el rendimiento en una prueba de memoria prospectiva de laboratorio en personas con discapacidad psíquica. Parecen claves para entender estos resultados discrepantes las diferencias entre las tareas utilizadas (de laboratorio o naturales), y la cantidad de incentivo empleada.

Los incentivos podrían estar facilitando el recuerdo prospectivo a través de un incremento de la motivación por realizar correctamente la tarea. En este sentido, la emoción implícita en la recompensa (satisfacción por conseguir un premio), está facilitando el recuerdo de realizar la conducta intencionada. La emoción influye en la conducta de muy diferentes maneras (Custers y Aarts, 2005; Damasio, 1994; Dreisbach y Goschke, 2004; Rolls, 2000), valga de ejemplo el modo en que lo hace a través de los incentivos. En el mismo sentido, a nivel social la emoción puede actuar como una señal de refuerzo o castigo de la conducta; es decir, como una vía de comunicación afectiva (Picard, 1997); por ejemplo, en la relación padre-hijo, una sonrisa puede consolidar una conducta, mientras que un gesto desaprobatorio puede inhibirla. De igual forma se puede interpretar que tras la realización de una conducta intencionada (e.g., ir al dentista), la generación del estado afectivo consecuente (e.g., positivo: alivio porque desapareció el dolor), podría facilitar o perjudicar el recuerdo de realizar dicha conducta la próxima vez que sea necesario.

Es decir, inducir un estado emocional tras la realización de una conducta intencionada, y hacerlo en una tarea de laboratorio, permitiría estudiar este supuesto. Con esta forma de enfocar la investigación se pueden conocer los efectos de la emoción de manera específica sobre uno de los componentes de la tarea prospectiva (conducta intencionada) evitando, en lo posible, perjudicar la validez ecológica, ya que en la vida cotidiana son habituales situaciones en las que un estado emocional aparece tras un hecho determinado, mientras que parece menos lógico pensar que en la población sin trastornos psiquiátricos como la depresión o la ansiedad, el estado afectivo se mantenga estable a lo largo del día. Planteamiento éste que ha sido aceptado en trabajos que inducen un estado emocional previo a la prueba con el fin de comprobar el efecto de éste sobre la MP (e.g., Kliegel et al., 2005), con la dificultad de que dicho estado no se mantiene constante a lo largo de la tarea (Kliegel y Jäger, 2006), lo que podría estar afectando al resultado final de manera no controlada.

Llegados a este punto, y entendiendo la emoción como una señal de comunicación social capaz de reforzar la conducta (Broekens, 2007), se puede plantear una hipótesis de trabajo que contemple la idea de que la inducción de una emoción (positiva o negativa) tras la realización de la conducta intencionada, podría estar "reforzando" o "castigando" la realización de dicha conducta en el futuro, afectando al recuerdo de recordar (MP). En definitiva, la emoción afecta a la conducta en diferentes aspectos y maneras, como lo demuestra el hecho de que la capacidad de tener sensaciones de placer y dolor está estrechamente relacionada con los mecanismos básicos del aprendizaje y la toma de decisiones (Berridge, 2003), lo que evidenciaría que las áreas importantes en la emoción, también lo son para el aprendizaje instrumental (Broekens, 2007).

\section{Refuerzo y castigo de la conducta intencionada}

Los trabajos consultados permiten proponer un nuevo enfoque de investigación, donde la conducta intencionada podría ser modulada mediante procedimientos operantes (refuerzo y castigo), que tendrían que ser aplicados durante la tarea de MP e inmediatamente después de la realización o no realización de la conducta intencionada (véase tabla 2). Al tratar con premios y castigos, se debe tener en cuenta que las conductas se consideran positivas o negativas, dependiendo de la emoción que generan sus consecuencias. Por ejemplo, la muerte de un animal nos causará una emoción negativa si se ha producido de manera accidental (se "vivenciará" como castigo), o positiva si se produce dentro de un contexto de caza (se "vivenciará" como refuerzo). En este caso, la interpretación cognitiva (accidente o 
caza) ha generado la emoción que definirá el valor hedónico o valencia de la conducta. Por lo tanto, al castigar o reforzar una conducta, estamos dando valor hedónico o valencia (positiva o negativa) a dicha conducta.

Tabla 2. Hipótesis sobre la aplicación de procedimientos operantes para modificar la frecuencia de aparición de la conducta intencionada.

\begin{tabular}{lcc}
\hline & \multicolumn{2}{c}{ Tipo de estímulo } \\
\hline & Positivo & Negativo \\
\hline Realizar la Conducta & Refuerzo & Castigo \\
Intencionada (Recuerdo) & $(>\mathrm{MP})$ & $(<\mathrm{MP})$ \\
No Realizar la Conducta & Refuerzo & Castigo \\
Intencionada (Olvido) & $(<\mathrm{MP})$ & $(>\mathrm{MP})$ \\
\hline Nota: $>$ MP: &
\end{tabular}

Ahora bien, ¿qué tipo de estímulo sería el más adecuado? En el paradigma del condicionamiento operante, cuando una rata aprieta una palanca y recibe comida, tanto la valencia positiva de la comida, como la activación que dicha comida provoca en el animal, repercutirán en el aprendizaje. Sin embargo, en tareas de memoria prospectiva no podemos presentar reforzadores primarios (e.g., comida) o secundarios (e.g., dinero), porque se transformaría en una tarea de atención dividida, donde el sujeto mantendría en mente de manera continuada a lo largo de la tarea la conducta a realizar para conseguir el premio o evitar el castigo. Se hace por tanto necesario inducir un estado emocional tras la realización o no realización de la conducta intencionada, sin que esto implique la presencia de un estímulo físico. Para este fin, se utilizaría el International Affective Picture System (IAPS; Lang, Bradley y Cuthbert, 1999), que es un conjunto estandarizado de fotografías a color, capaz de generar emociones y considerado el sistema más fiable y valido para el estudio experimental de las emociones (Jayaro, de la Vega, Díaz-Marsá, Montes y Carrasco, 2008). Este sistema permite inducir estados afectivos positivos (refuerzo), neutros o negativos (castigo) con la presentación de fotografías agradables, neutras o desagradables respectivamente, de manera contingente a la conducta intencionada, produciendo en el sujeto una sensación equiparable a la producida tras la presentación de un reforzador primario o secundario (e.g., alegría surgida al conseguir dinero o alimento), pero sin la presencia física de éste. Además, al estar las fotografías del IAPS clasificadas por sus niveles de arousal y valencia, permitiría un análisis mucho más detallado y nunca antes realizado del efecto de la emoción sobre la MP, y daría lugar a un planteamiento de hipótesis determinadas a partir de supuestos operantes (ver tabla 2).

Una de las dificultades principales a la hora de plantear una tarea de MP donde la realización o no realización de la conducta intencionada lleve asociada la presentación de una fotografía de contenido emocional, es la posibilidad de que la fotografía se asocie a la clave de recuperación, en tanto los resultados podrían explicarse entonces por la mayor o menor saliencia que las características de la fotografía generan sobre el estímulo que advierte del momento en el que debe realizarse la conducta intencionada (clave de recuperación).
Para solventar esta dificultad e implementar metodológicamente el planteamiento teórico desarrollado en este trabajo, se propone una tarea de memoria prospectiva que admite la inducción emocional durante la prueba, al mismo tiempo que mantiene las condiciones necesarias para la medida de un proceso intencional: a los sujetos se les presentan dibujos formados por dos figuras geométricas, una dentro de la otra. Los dibujos, de uno en uno, pueden aparecer en 9 diferentes localizaciones de la pantalla, y se mantienen hasta que se responda según la siguiente regla de clasificación. Deberán pulsar lo más rápido que puedan " 1 " cuando el dibujo tenga un triangulo dentro, " 2 " cuando sea un círculo y "3" un cuadrado. De esta forma queda configurada la tarea de fondo. Tras la realización de cada ensayo se les muestra una pantalla durante 2 segundos (feedback informativo), en la que se informa de lo "correcto" o "incorrecto" de la respuesta, junto al tiempo empleado y el porcentaje de respuestas correctas realizadas hasta ese momento.

También se les dirá que cuando el dibujo aparezca en la misma localización de la pantalla en la que apareció el dibujo anterior, no deben aplicar la regla clasificatoria sino pulsar " 0 " lo más rápido posible. De esta forma se configura la conducta intencionada; es decir, la acción programada en las instrucciones (pulsar "0") y que debe realizarse durante la tarea de fondo cuando aparezca la clave de recuperación (cuando el dibujo aparezca en la misma localización de la pantalla en la que apareció el dibujo anterior).

Por último, la fotografías del IAPS se presentan tras la realización o no realización de la conducta intencionada. El tipo de fotografía; la frecuencia de aparición y el tiempo de exposición vendrán determinados por los objetivos de la investigación.

Por lo tanto, la prueba se presenta como una tarea de tiempo de reacción en la que la conducta intencionada es una tarea secundaria, y donde la clave de recuperación no es un estímulo determinado sino una sucesión de dos estímulos (tienen que aparecer dos dibujos, sean cuales sean, de manera consecutiva en la misma localización de la pantalla), esto permite delimitar el proceso intencional, en el sentido de que para realizar una conducta intencionada, el sujeto debe tener activada la intención, por lo menos, desde el ensayo anterior a la realización de la conducta intencionada (apretar “0”), de otra forma el sujeto no podrá saber si el dibujo apareció o no en la misma posición que el anterior. El hecho de que la clave de recuperación no sea un tipo de estímulo determinado evita que la fotografía del IAPS se asocie a dicho estimulo y le suministre una saliencia que permitiría explicar los resultados por la alta demanda atencional adquirida por dicho estímulo.

\section{Conclusiones}

La capacidad para almacenar experiencias y beneficiarse de ellas en un futuro resulta de vital importancia para el ser humano (Ballesteros, 1999). De igual manera resulta vital la capacidad de planificación de nuestras acciones, y sobre todo la 
capacidad de recordar realizar dichas acciones en un momento determinado del futuro. Dentro de nuestra sociedad, la correcta planificación del tiempo es importante para lograr compatibilizar trabajo y vida familiar. Esto resulta complicado porque el alto grado de información al que estamos sometidos dificulta el adecuado procesamiento atencional, necesario para el recuerdo de realizar estas acciones programadas. Quizá olvidarse de comprar el pan debido a la gran cantidad de requerimientos que hemos atendido durante el día, no tenga consecuencias muy graves, pero este tipo de olvidos en las personas mayores genera un alto grado de malestar, ya que son percibidos como situaciones muy debilitantes para el adecuado desempeño diario y provocan un deterioro de la autoestima (Meyer y Mattos, 2003).

En este contexto, como en todas las situaciones en las que esté involucrado un ser humano, la emoción está presente, y el objetivo de este trabajo ha sido el de entender en qué sentido afecta al procesamiento intencional, y cómo sería posible su medida de manera fiable. En conclusión, la emoción influye de manera diferencial sobre los principales componentes de la tarea de MP (tarea de fondo, conducta intencionada, claves de recuperación), y al inducir una emoción tras la realización o no realización de la conducta intencionada, se está utilizando la emoción como una vía de comunicación afectiva (Picard, 1997) que permitiría modular dicha conducta y por lo tanto determinar, en un sentido todavía no estudiado, la tasa de memoria prospectiva.

En futuras investigaciones, una vez comprobado el posible efecto de inducir una emoción tras la realización o no realización de la conducta intencionada sobre la MP, resultaría interesante comprobar si la frecuencia y periodo de exposición de las fotografías utilizadas para la inducción emocional, podrían estar mediando en la modulación de la conducta intencionada. Este estudio resultaría de especial relevancia porque permitiría comprender con qué duración y frecuencia debemos aplicar un castigo para promover una mejora en la memoria de intenciones: ¿Cómo debemos presentar los castigos o refuerzos al niño para que sean más efectivos en la planificación de su conducta futura? ¿Cómo se debe plantear la estructura de una anuncio de prevención de riesgos sociales (e.g., tráfico, sexualidad, drogadicción) para que tengan la mayor eficacia posible sobre el recuerdo de las consecuencias de no evitar un tipo de conducta determinado (e.g., subirse al coche bebido, tener relaciones sexuales sin protección, probar por primera vez una droga). De igual manera la utilización de otras bases de estímulos como palabras (ANEW; Bradley y Lang, 1999a) y sonidos (IADS; Bradley y Lang, 1999b) en el proceso de inducción emocional, aportarían mayor solidez a la generalización de los resultados.

Por último, tener en cuenta que las conductas intencionadas de la vida real con frecuencia poco tienen que ver con las realizadas en laboratorio; sin embargo, existe una base teórica común que sustenta a unas y otras y que supone un continuo de lo básico a lo aplicado que debe tenerse en cuenta a la hora de elaborar una prueba de este tipo. Si además se implica a las emociones, entonces se hace necesario delimitar muy claramente el rango de estudio y aplicar pruebas estandarizadas para su medida. Este trabajo plantea un nuevo enfoque de investigación que demanda un marco teórico que integre en el estudio de la MP conceptos presentes en el paradigma del condicionamiento instrumental.

\section{Referencias}

Adda, C. C., Castro, L. H., Além-Mar e Silva, L. C., de Manreza, M. L. y Kashiara, R. (2008). Prospective memory and mesial temporal epilepsy associated with hippocampal sclerosis. Neuropsychologia 46(7), 1954-1964.

Altgassen, M., Kliegel, M. y Martin, M. (2009). Event-based prospective memory in depression: The impact of cue focality. Cognition and Emotion, 23(6), 1041-1055.

Altgassen, M., Phillips, L. H., Henry, J. D., Rendell P. G. y Kliegel, M. (2010). Emotional target cues eliminate age differences in prospective memory. The Quarterly Journal of Experimental Psychology, 63(6), 1057-1064.

Arana, J. M., Gordillo, F., Meilán, J. J. G., Carro, J., Mestas, L. y Salvador, J. (2010, julio). Negative emotion facilitating effect on prospective memory. Póster presentado en Simposio del 3Rd International Conference on Prospective Memory. University of British Columbia. Vancouver, Canada.

Ballesteros, S. (1999). Memoria humana: investigación y teoría. Psicothema, 11(4), 705-723.

Basso, D., Ferrari, M. y Palladino, P. (2010). Prospective memory and working memory: Asymmetrical effects during frontal lobe TMS stimulation. Neuropsychologia, 48(11), 3282-3290.

Berridge, K. C. (2003). Pleasures of the brain. Brain and Cognition 52(10), 106-128.

Bradley, M. M. y Lang, P. J. (1999a). Affective norms for English words (ANEW): Stimuli, instruction manual and affective ratings. Technical report C-1. Gainesville, FL. The Center for Research in Psychophysiology, University of Florida.

Bradley, M. M. y Lang, P. J. (1999b). International affective digitized sounds (IADS): Stimuli, instruction manual and affective ratings. Technical report B-2. Gainesville, FL: The Center for Research in Psychophysiology, University of Florida.

Broekens, J. (2007). Emotion and Reinforcement: Affective facial expressions facilitate robot learning. Lecture Notes in Computer Science, 4451, 113-132.

Chan, R. C. K., Wang, Y., Ma, Z., Hong, X-H., Yuan, Y., Yu, X., Li, A., Shum, D., Gong, Q-H. (2008). Objective measures of prospective memory do not correlate with subjective complaints in schizophrenia. Schizophrenia Research, 103(1), 229-239.

Clark-Foos, A., Brewer, G. A. y Marsh, R. L. (2009). The va- 
lence of event-bases prospective memory cues or the context in which they occur affects their detection. American Journal of Psychology, 122(1), 89-97.

Costa, A., Peppe, A., Caltagirone, C. y Carlesimo, G. A. (2008). Prospective memory impairment in individuals with Parkinson's disease. Neuropsychology 22(3), 283-292.

Custers, R. y Aarts, H. (2005). Positive affect as implicit motivator: On the nonconscious operation of behavioral goals. Journal of Personality and Social Psychology, 89(2), 129142.

Damasio, A. R. (1994). Descartes' error. New York, NY: Penguin Putnam.

Dreisbach, G. y Goschke, T. (2004). How positive affect modulates cognitive control: Reduced perseveration at the cost of increased distractibility. Journal of Experimental Psychology: Learning, Memory, and Cognition, 30(2), 343-353.

Einstein, G. O. y McDaniel, M. A. (1996). Retrieval processes in prospective memory: Theoretical approaches and some new empirical findings. En M. A. Brandimonte, G. O. Einstein y M. A. McDaniel (Eds.), Prospective memory: Theory and application. Mahwah, NJ: Lawrence Erlbaum Associates.

Fernández-Rey, J. y Redondo, J. (2007). Recognition memory for pictorial stimuli: Biasing effects of stimulus emotionality. Psicothema, 19(3), 375-380.

Fish, J., Wilson, B. A. y Manly, T. (2010). The assessment and rehabilitation of prospective memory problems in people with neurological disorders: A review. Neuopsychological Rehabilitation, 20(2), 161-179.

Fleming, J. M., Shum, D., Strong, J. y Lightbody, S. (2005). Prospective memory rehabilitation for adults with traumatic brain injury: A compensatory training programme. Brain Injury, 19(1), 1-10.

Foster, E. R., McDaniel, M. A., Repovŝ, G. y Hershey, T. (2009). Prospective memory in Parkinson disease across laboratory and self-reported every day performance. $\mathrm{Neu}$ ropsychology, 23(3), 347-358.

Glisky, E. L. (1996). Prospective memory and the frontal lobes. En: Brandimonte M, Einstein G. O., McDaniel M. A., Eds. Prospective memory: theory and application (pp. 249-266). Mahwah, NJ: Lawrence Erlbaum.

Gordillo, F., Arana J. M., Mestas L., Salvador, J., Meilán J. J. G., Carro, J. y Pérez, E. (2010). Emoción y memoria de reconocimiento: la discriminación de la información negativa como un proceso adaptativo. Psicothema, 22(4), 765-771.

Graff, P. y Uttl, B. (2001). Prospective memory: A new focus for research. Consciousness and Cognition 10(4), 437-450.

Guajardo, N. R. y Best, D. L. (2000). Do preschoolers remember what to do? Incentive and external cues in prospective memory. Cognitive Development, 15(1), 75-97.

Harris, L. M. y Cumming, S. R. (2003). An examination of the relationship between anxiety and performance on prospective and retrospective memory tasks. Australian Journal of
Psychology, 55(1), 51-55.

Harris, L. M. y Menzies, R. G. (1999). Mood and prospective memory. Memory, 7(1), 117-127.

Heffernan, T., Clark, R., Bartholomew, J., Ling, J. y Stephens, S. (2010). Does binge drinking in teenagers affect their everyday prospective memory? Drug and Alcohol Dependence, 109(1-3), 73-78.

Henry, J. D., MacLeod, M. S., Phillips, L. H. y Crawford, J. R. (2004). A meta-analytic review of prospective memory and aging. Psychology and Aging, 19(1), 27-39.

Jayaro, C., de la Vega, I., Díaz-Marsá, M., Montes, A. y Carrasco, J. L. (2008). Aplicaciones del International Affective Picture System en el estudio de la regulación emocional en los trastornos mentales. Actas Españolas de Psiquiatría, 36(3), 177-182.

Kliegel, M., McDaniel, M. A. y Einstein G. O., (2008). Prospective Memory. Cognitive Neuroscience, Developmental, and Applied Perspectives. New York: Lawrence Erlbaum Associates, Taylor- Francis Group.

Kliegel, M. y Jäger, Th. (2006). The influence of negative emotions on prospective memory: a review and new data (invited paper). International Journal of Computational Cognition, 4(1), 1-17.

Kliegel, M., Jäger, T., Phillips, L. H., Federspiel, E., Imfeld, A., Keller, M. y Zimprich, D. (2005). Effects of sad mood on time-based prospective memory. Cognition y Emotion, 19(8), 1199-1213.

Kuhl, J. y Kazén, M. (1999). Volitional facilitation of difficult intentions: Joint activation of intention memory and positive affect removes Stroop interference. Journal of Experimental Psychology: General, 128(3), 382-399.

Kvavilashvili, L. (1987). Remembering intentions as a distinct form of memory. British Journal of Psychology, 78, 507518.

Kvavilashvili, L. (1992). Remembering intentions: A critical review of existing experimental paradigms. Apllied Cognitive Psychology, 6(6), 507-524.

Kvavilashvili, L. y Ellis, J. (1996). Varieties of intentions: Some distinctions and classifications. En M. Brandimonte, G. O. Einstein., y M. A. McDaniel (Eds.), Prospective memory: Theory and applications (pp. 23-51). Mahwah, NJ: Erlbaum.

Lang, P. J., Bradley, M. M. y Cuthbert, B. N. (1999). International affective picture system (IAPS): Technical manual and affective ratings. Gainesville, FL: The Center for Research in Psychophysiology, University of Florida.

Livner, A., Laukka, E. J., Karlsson, S. y Bäckman, L. (2009). Prospective and retrospective memory in Alzheimer's disease and vascular dementia: Similar patterns of impairment. Journal of the Neurological Sciences 283(1-2), 235-239.

Matter, S. y Meier, B. (2008). Prospective memory affects satisfaction with the contraceptive pill. Contraception, 78(2), 120-124. 
McFarland, C. P. y Glisky, E. L. (2009). Frontal lobe involvement in a task of time-based prospective memory. Neuropsychologia, 47(7), 1660-1669.

Meacham, J. A. y Kushner, S. (1980). Anxiety, prospective remembering and performance of planned actions. Journal of General Psychology, 103(2d Half), 203-209.

Meacham, J. A. y Leiman, B. (1982). Remembering to perform future actions. En U. Neisser (Ed.), Memory observed: Remembering in natural contexts (pp. 327-336). San Francisco: Freeman.

Meacham, J. A. y Singer, J. (1977). Incentive effects in prospective remembering. The Journal of Psychology, 97, 191-197.

Meilán, J. J. G., Pérez, E. y Arana, J. M. (2005). Procesos cognitivos y neurológicos implicados en la memoria intencional. Revista Electrónica de Motivación y Emoción, 20 (extraído el 21 de agosto de 2010 en http://reme.uji.es/).

Meilán, J. J. G., Pérez, E., Arana, J. M. y Valles, J. M. (2006). Estudio de la memoria prospectiva en personas con discapacidad psíquica: implicaciones de la motivación y de los factores neuropsicológicos. Infancia y Aprendizaje, 29(2), 137-146.

Meilán, J. J. G., Salgado, V. M., Arana, J. M., Carro, J. y Jenaro, C. (2008). Entrenamiento cognitivo y mejora de la memoria prospectiva en jóvenes con retraso mental leve. Revista de Investigación Educativa, 26(1), 227-245.

Meyer, I. y Mattos, M. A. (2003). La vulnerabilidad de memoria prospectiva en las quejas de memoria del adulto. Revista Neurológica Argentina, 28, 85-90.

Oaksford, M., Morris, F., Grainger, B. y Williams, J. M. G. (1996). Mood, reasoning, and central executive processes. Journal of Experimental Psychology: Learning, Memory, and Cognition, 22(2), 477-493.

Phillips, L. H., Smith, L. y Gilhooly, K. J. (2002). The effects of adult aging and induced positive and negative mood on planning. Emotion, 2(3), 263-272.

Picard, R. W. (1997). Affective Computing. Cambridge, MA: The MIT Press.

Redondo, J. y Fernández-Rey, J. (2010). Reconocimiento de fotografías de contenido emocional: Efectos de la valencia cuando se controla el arousal. Psicológica, 31, 65-86.

Reynolds, J. R., West, R. y Braver, T. (2009). Distinct neural circuits support transient and sustained processes in prospective memory and working memory. Cerebral Cortex 19(5), 1208-1221.

Rolls, E. T. (2000). Précis of The brain and emotion. Behavioral and Brain Sciences, 23(2), 177-191.

Rude, S. S., Hertel, P. T., Jarrold, W., Covich, J. y Hedlund, S. (1999). Depression-related impairments in prospective memory. Cognition and Emotion, 13(3), 267-276.

Schmidt, S. R. (2004). Autobiographical memories for the September 11th attacks: Reconstructive errors and emotional impairment of memory. Memory and Cognition, 32(3), 443-454.
Schmidt, I. W., Berg, I. J. y Deelman, B. G. (2001). Prospective memory training in older adults. Educational Gerontology, 27(6), 455-478.

Shallice, T. y Burgess, P. W. (1991). Deficits in strategy application following frontal lobe damage in man. Brain 114(Pt 2), 727-741.

Shimamura, A. P., Janowsky, J. S. y Squire, L. R. (1991). What is the role of frontal lobe damage in amnesic disorders? En H. S. Levin, H. M. Eisenberg, y A. L. Benton (Eds.), Frontal Lobe Functioning and Dysfunction. Oxford University Press: Oxford.

Simón, T., Ruiz, T. y Suengas, A. G. (2009). Memoria y envejecimiento: recuerdo, reconocimiento y sesgo positivo. Psicothema, 21(3), 409-415.

Simons, J. S., Scholvinck, M. L., Gilbert, S. J., Frith, C. D. y Burgess, P. W. (2006). Differential components of prospective memory? Evidence from fMRI. Neuropsychologia 44(8), 1388-1397.

Sohlberg, M. M., White, O., Evans, E. y Mateer, C. (1992). Background and initial case studies into the effects of prospective memory training. Brain Injury, 6(2), 129-138.

Somerville, S. C., Wellman, H. M. y Cultice, J. C. (1983). Young children's deliberate reminding. Journal of Genetic Psychology, 143, 87-96.

Twamley, E. W., Woods, S. P., Zurhellen, C. H., Vertinski, M., Narvaez, J. M., Mausbach, B. T., Patterson, T. L. y Jeste, D. V. (2008). Neuropsychological substrates and everyday functioning implications of prospective memory impairment in schizophrenia. Schizophrenia Research 106(1), 42-49.

Umeda, S., Nagumo, Y. y Kato, M. (2006). Dissociative contributions of medial temporal and frontal regions to prospective remembering. Reviews in the Neurosciences. 17(1-2), 267-278.

Ungvari, G. S., Xiang, Y-T., Tang, W-K. y Shum, D. (2008). Prospective memory and its correlates and predictors in schizophrenia: An extension of previous findings. Archives of Clinical Neuropsychology, 23(5), 613-622.

Wang, Y., Chan, R. C. K., Cui, J., Deng, Y., Huang, J., Li, H., Yan, C., Xu, T., Ma, Z., Hong, X., Li, Z., Shi, H. y Shum, D. (2010). Prospective memory in non-psychotic first-degree relatives of patients with schizophrenia. Psychiatry Research, 179(3), 285-290.

Woods, S. P., Moran, L. M., Carey, C. L., Dawson, M. S., Iudicello, J. E., Gibson, S., Grant I, Atkinson JH; HIV Neurobehavioral Research Center Group. (2008). Prospective memory in HIV infection: Is "remembering to remember" a unique predictor of self-reported medication management? Archives of Clinical Neuropsychology, 23(3), 257-270.

Fecha de recepción: 5 de octubre de 2010

Fecha de recepción de la primera versión modificada: 18 de diciembre de 2010 Fecha de aceptación: 21 de diciembre de 2010 